\title{
A General Method for the Enantioselective Synthesis of Pantolactone Derivatives
}

\author{
David A. Evans*, Jimmy Wu, Craig E. Masse, and David W. C. MacMillan \\ Department of Chemistry \& Chemical Biology, Harvard University, Cambridge, Massachusetts 02138
}

Supporting Information

General Information. All reactions were carried out under an atmosphere of argon or nitrogen in ovendried glassware with magnetic stirring. THF and $\mathrm{CH}_{2} \mathrm{Cl}_{2}$ were purified by passage through a bed of activated alumina. ${ }^{1}$ Solvents and reagents were purified prior to use following the guidelines of Perrin and Armarego. ${ }^{2} \mathrm{ScCl}_{3} \mathrm{thf}_{3}$ was prepared according to literature procedure. ${ }^{3} \mathrm{AgSbF}_{6}$ was purchased from the Cerac Chemical Co., stored in an inert atmosphere dry box and used without further purification. $\mathrm{Me}_{4} \mathrm{NHB}(\mathrm{OAc})_{3}$ was prepared according to literature procedure. ${ }^{4} \mathrm{Et}_{2} \mathrm{BOMe}$ was purchased from Aldrich Chemical Co. and used as is after distillation. Purification of reaction products was carried out by flash chromatography using EM Reagent silica gel 60 (230-400 mesh). Analytical thin layer chromatography was performed on EM Reagent $0.25 \mathrm{~mm}$ silica gel $60-\mathrm{F}$ plates. Visualization was accomplished with UV light and ceric ammonium nitrate stain followed by heating. Optical rotations were measured on a Jasco DIP-0181 digital polarimiter with a sodium lamp and are reported as follows: $[\alpha]_{\lambda}{ }^{\mathrm{T}}{ }^{\circ} \mathrm{C}(c \mathrm{~g} / 100 \mathrm{~mL}$, solvent). Infrared spectra were recorded on a Perkin Elmer 1600 series FT-IR spectrometer. ${ }^{1} \mathrm{H}$ NMR spectra were recorded on a Varian Inova-500 (500 MHz) or Varian Mercury$400(400 \mathrm{MHz})$ spectrometer and are reported in ppm using solvent as an internal standard $\left(\mathrm{CDCl}_{3}\right.$ at $7.26 \mathrm{ppm}$ ). Data reported as ( $\mathrm{s}=$ singlet, $\mathrm{d}=$ doublet, $\mathrm{t}=$ triplet, $\mathrm{q}=$ quartet, $\mathrm{m}=$ multiplet, $\mathrm{b}=$ broad; integration; coupling constant(s) in $\mathrm{Hz}$ ). Proton-decoupled ${ }^{13} \mathrm{C}$ NMR spectra were recorded on Varian Inova-500 (125 MHz) or Varian Mercury-400 (100 MHz) spectrometer and are reported in ppm using solvent as an internal standard $\left(\mathrm{CDCl}_{3}\right.$ at $\left.77.0 \mathrm{ppm}\right)$. High resolution mass spectra were obtained on Jeol AX-505 or SX-102 spectrometers at the Harvard University Mass Spectrometry Laboratory. Analytical high performance liquid chromatography (HPLC) was performed on a Hewlett-Packard 1100 Series HPLC with a diode array detector using the indicated chiral column.

General Procedure for the Catalyzed Reaction of Thiosilylketene Acetals with Ethyl Glyoxylate. A dry flask was charged with $\mathrm{ScCl}_{3}(\mathrm{thf})_{3}(0.10$ equiv) and phenyl-pyridyl-bis(oxazolinyl) ligand $(0.11$ equiv) in an inert atmosphere $\left(\mathrm{N}_{2}\right)$ glove box. In a separate flask, also in an inert atmosphere $\left(\mathrm{N}_{2}\right)$ glove box, was charged $\mathrm{AgSbF}_{6}$ (0.9 equiv). Both flasks were brought out of the glove box and the flask containing the metal/ligand was charged with $\mathrm{CH}_{2} \mathrm{Cl}_{2}(2 \mathrm{~mL})$ and stirred at $\mathrm{rt}$ for 30 minutes. The resulting solution was transferred to the flask containing $\mathrm{AgSbF}_{6}$ via syringe and the mixture stirred for 30 minutes followed by cooling to $-78^{\circ} \mathrm{C}$. Ethyl glyoxylate (1.5 equiv) and thiosilylketene acetal (1.0 equiv) were added sequentially and stirred for $3 \mathrm{~h}$ at $-78{ }^{\circ} \mathrm{C}$. After the reaction was complete, the mixture was filtered through silica with $\mathrm{Et}_{2} \mathrm{O}$ and the silyl ether was hydrolyzed with $1 \mathrm{~N} \mathrm{HCl}$ in EtOAc ( $30 \mathrm{~min}, 25^{\circ} \mathrm{C}$ ) to give the hydroxy ester which was purified by flash chromatography. ${ }^{5}$

General Procedure for the Catalyzed Reaction of Enolsilanes with Ethyl Glyoxylate. A dry flask was charged with $\mathrm{ScCl}_{3}(\text { thf })_{3}$ (0.15 equiv) and $t$-Bu-pyridyl-bis(oxazolinyl) ligand (0.17 equiv) in an 
inert atmosphere $\left(\mathrm{N}_{2}\right)$ glove box. In a separate flask, also in an inert atmosphere $\left(\mathrm{N}_{2}\right)$ glove box, was charged $\mathrm{AgSbF}_{6}(0.14$ equiv). Both flasks were brought out of the glove box and the flask containing the metal/ligand was charged with $\mathrm{CH}_{2} \mathrm{Cl}_{2}(1 \mathrm{~mL})$ and stirred at $\mathrm{rt}$ for $1 \mathrm{~h}$. The resulting solution was transferred to the flask containing $\mathrm{AgSbF}_{6}$ via syringe and the mixture stirred for 30 minutes followed by cooling to $-78^{\circ} \mathrm{C}$. Ethyl glyoxylate (1.5 equiv), enolsilane (1.0 equiv), and TMS-Cl ( 2 equiv) were added sequentially and the reaction stirred for $16 \mathrm{~h}$ at $-35^{\circ} \mathrm{C}$. After the reaction was complete, the mixture was filtered through silica with $\mathrm{Et}_{2} \mathrm{O}$ and the silyl ether was hydrolyzed with $1 \mathrm{~N} \mathrm{HCl}$ in EtOAc ( $30 \mathrm{~min}, 25^{\circ} \mathrm{C}$ ) to give the hydroxy ester which was purified by flash chromatography. ${ }^{5}$

General Procedure (A): Raney Nickel Reduction/Cyclization of Thioesters to Pantolactones. To a round bottom flask containing a magnetic stirring bar, was added isopropanol $(1.0 \mathrm{~mL})$ and Raney $\mathrm{Ni}$ ( $1.0 \mathrm{~mL}$ of a slurry in $\mathrm{H}_{2} \mathrm{O}, 0.5 \mathrm{~g}$ catalyst $/ \mathrm{mL}$ of settled material). This mixture was purged with hydrogen from a balloon under vigorous stirring. To this suspension of Raney $\mathrm{Ni}$, was added a solution of the aldol adduct (1.0 equiv) in $\mathrm{Et}_{2} \mathrm{O}(0.52 \mathrm{~mL}, 0.25 \mathrm{M})$. The reaction mixture was stirred vigorously until the aldol adduct was completely consumed (15-30 $\mathrm{min})$, as determined by TLC (30\% EtOAc/hexanes). The reaction mixture was then filtered through a pad of Celite and washed repeatedly with EtOH $(3 \times 25 \mathrm{~mL})$. The filtrate was concentrated in vacuo to provide the unpurified lactone. Purification by flash chromatography (30-40\% EtOAc/hexanes) provided the title compounds.

General Procedure (B): Syn Reduction/Cyclization of Hydroxy-Esters to Pantolactones. To an oven dried flask was added $\mathrm{Me}_{4} \mathrm{NHB}(\mathrm{OAc})_{3}$ (8.4 equiv) and $\mathrm{MeCN} / \mathrm{AcOH}(1: 1 \mathrm{v} / \mathrm{v})$ to afford a $0.9 \mathrm{M}$ solution which was stirred for $30 \mathrm{~min}$ at $\mathrm{rt}$ before cooling to $-20{ }^{\circ} \mathrm{C}$. To this mixture, was added a solution of hydroxy-ester $\left(1.0\right.$ equiv) in $\mathrm{MeCN}(0.65 \mathrm{M})$ and aged for $1 \mathrm{hr}$ at $-20{ }^{\circ} \mathrm{C}$ before warming to $0{ }^{\circ} \mathrm{C}$ and aging for an additional $16 \mathrm{hr}$. To the reaction was added sat. Rochelle's salt and sat. $\mathrm{NaHCO}_{3}$ and then extracted with with $\mathrm{CH}_{2} \mathrm{Cl}_{2}(4 \mathrm{x} 10 \mathrm{~mL})$. The organic layers were combined and washed with $\mathrm{NaHCO}_{3}(10 \mathrm{~mL})$. The aqueous layer was back-extracted with $\mathrm{CH}_{2} \mathrm{Cl}_{2}(4 \times 10 \mathrm{~mL})$. The organic layers were combined and concentrated in vacuo to afford a colorless oil which was transferred to a dry flask and charged with benzene $(0.23 \mathrm{~mL}), 3 \AA \mathrm{MS}(25 \mathrm{mg})$, and $p$-TSA ( 0.5 equiv). The reaction was stirred at $\mathrm{rt}$ for $30 \mathrm{~min}$. and diluted with $\mathrm{Et}_{2} \mathrm{O}(2 \mathrm{~mL})$. Triethylamine (6 drops) was added to the mixture which was then filtered through a plug of silica gel and flushed with $\mathrm{Et}_{2} \mathrm{O}(50 \mathrm{~mL})$. Concentration of the solvent in vacuo provided the desired product analytically pure.

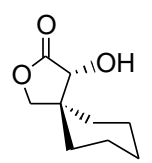

(R)-4-Hydroxy-2-oxa-spiro[4.5]decan-3-one (Table 2, entry 1). The title compound was prepared according to General Procedure A and isolated as a colorless oil (89\% yield): $[\alpha]_{\mathrm{D}}{ }^{25}$ $+16.3^{\circ}\left(c 0.5, \mathrm{CHCl}_{3}\right)$; IR (film) 3442, 2938, 2860, 1762, 1559, 1473, 1164, 1103, $990 \mathrm{~cm}^{-1}$; ${ }^{1} \mathrm{H}$ NMR $\left(400 \mathrm{MHz}, \mathrm{CHCl}_{3}\right) \delta 4.33(\mathrm{~d}, 1 \mathrm{H}, J=9.2 \mathrm{~Hz}), 4.10(\mathrm{~d}, 1 \mathrm{H}, J=4.3 \mathrm{~Hz}), 3.87(\mathrm{dd}, 1 \mathrm{H}$, $J=1.4,9.2 \mathrm{~Hz}), 3.49(\mathrm{~d}, 1 \mathrm{H}, J=4.4 \mathrm{~Hz}), 1.77-1.54(\mathrm{~m}, 6 \mathrm{H}), 1.44-1.35(\mathrm{~m}, 2 \mathrm{H}), 1.26-1.16(\mathrm{~m}, 2 \mathrm{H}) ;{ }^{13} \mathrm{C}$ NMR $\left(100 \mathrm{MHz} \mathrm{CHCl}_{3}\right) \delta 178.0,75.6,73.7,44.0,33.6,25.8,25.3,22.9,21.8 ; \mathrm{HRMS}\left(\mathrm{CI}, \mathrm{NH}_{3}\right)$ : Exact mass calcd for $\mathrm{C}_{8} \mathrm{H}_{12} \mathrm{O}_{3}\left[\mathrm{M}+\mathrm{NH}_{4}\right]^{+}, 188.1287$. Found 188.1283.

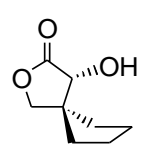

(R)-4-Hydroxy-2-oxa-spiro[4.4]nonan-3-one (Table 2, entry 2). The title compound was prepared according to General Procedure A and isolated as a colorless oil (94\% yield): $[\alpha]_{\mathrm{D}}{ }^{25}$ $+19.3^{\circ}\left(c 1.5, \mathrm{CHCl}_{3}\right)$; IR (film) 3390, 2952, 2869, 1768, 1318, 1289, 1201, 1146, 1100, 992 $\mathrm{cm}^{-1} ;{ }^{1} \mathrm{H} \mathrm{NMR}\left(400 \mathrm{MHz}, \mathrm{CHCl}_{3}\right) \delta 4.33(\mathrm{~d}, 1 \mathrm{H}, J=3.9 \mathrm{~Hz}), 4.12(\mathrm{~d}, 1 \mathrm{H}, J=8.8 \mathrm{~Hz}), 4.00$ (dd, 
$1 \mathrm{H}, J=0.9,8.8 \mathrm{~Hz}), 3.38$ (br, 1H), 2.03-1.96 (m, 1H), 1.93-1.86 (m, 1H), 1.80-1.58 (m, 5H), 1.44-1.38 $(\mathrm{m}, 1 \mathrm{H}) ;{ }^{13} \mathrm{C} \mathrm{NMR}\left(100 \mathrm{MHz}, \mathrm{CHCl}_{3}\right) \delta 177.8,76.0,73.6,51.6,33.5,29.1,25.0,24.9$; HRMS (CI, $\mathrm{NH}_{3}$ ): Exact mass calcd for $\mathrm{C}_{8} \mathrm{H}_{12} \mathrm{O}_{3}\left[\mathrm{M}+\mathrm{NH}_{4}\right]^{+}, 174.1130$. Found 174.1125.

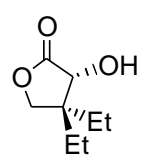

(R)-4,4-Diethyl-3-hydroxy-dihydro-furan-2-one (Table 2, entry 3). The title compound was prepared according to the General Procedure A to provide the pure product as a light yellow oil (95\% yield) after flash chromatography $40 \%$ EtOAc/hexanes: $[\alpha]_{\mathrm{D}}{ }^{25}-12.0^{\circ}(c$ $0.33, \mathrm{MeOH})$; IR (neat) 3423, 2976, 1754, 1637, $1461 \mathrm{~cm}^{-1} ;{ }^{1} \mathrm{H} \mathrm{NMR}\left(500 \mathrm{MHz}, \mathrm{CDCl}_{3}\right) \delta$ $4.21(\mathrm{br} \mathrm{d}, 1 \mathrm{H}, J=2.8 \mathrm{~Hz}), 4.16(\mathrm{~d}, 1 \mathrm{H}, J=8.8 \mathrm{~Hz}), 3.87(\mathrm{~d}, 1 \mathrm{H}, J=9.2 \mathrm{~Hz}), 2.55(\mathrm{br} \mathrm{d}, 1 \mathrm{H}, J=2.4$ $\mathrm{Hz}), 1.65-1.42(\mathrm{~m}, 4 \mathrm{H}), 0.98(\mathrm{t}, 3 \mathrm{H}, J=8.0 \mathrm{~Hz}), 0.90(\mathrm{t}, 3 \mathrm{H}, J=7.6 \mathrm{~Hz}) ;{ }^{13} \mathrm{C} \mathrm{NMR}\left(125 \mathrm{MHz}, \mathrm{CDCl}_{3}\right)$ $\delta$ 178.2, 74.8, 73.1, 46.7, 28.1, 21.4, 8.49, 8.05; HRMS (CI/NH3) exact mass calcd for $\mathrm{C}_{8} \mathrm{H}_{15} \mathrm{O}_{3}$ $[\mathrm{M}+\mathrm{H}]^{+}, 159.1021$. Found 159.1019. Assay of enantiomeric excess: GC analysis ( $\beta$-Cyclodextrin, 60 ${ }^{\circ} \mathrm{C}, 3{ }^{\circ} \mathrm{C} / \mathrm{min}$ at $25 \mathrm{psi} ; \mathrm{t}_{\mathrm{r}}($ major $)=24.38, \mathrm{t}_{\mathrm{r}}($ minor $\left.)=24.0\right), 95 \%$ ee.

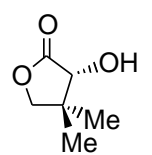

(R)-3-Hydroxy-4,4-dimethyl-dihydro-furan-2-one (Table 2, entry 4). The title compound was prepared according General Procedure A to provide the pure product as a light yellow oil (95\% yield) after flash chromatography with $30 \%$ EtOAc/hexanes: $[\alpha]_{\mathrm{D}}{ }^{25}-54.0{ }^{\circ}(c 0.25$, $\mathrm{H}_{2} \mathrm{O}$ ); IR (neat) 3442, 2092, 1758, 1638, $1124 \mathrm{~cm}^{-1} ;{ }^{1} \mathrm{H}$ NMR $\left(500 \mathrm{MHz}, \mathrm{CDCl}_{3}\right) \delta 4.11$ (br s, $\left.1 \mathrm{H}\right), 4.03$ $(\mathrm{d}, 1 \mathrm{H}, J=9.0 \mathrm{~Hz}), 3.94(\mathrm{~d}, 1 \mathrm{H}, J=8.5 \mathrm{~Hz}), 1.24(\mathrm{~s}, 3 \mathrm{H}), 1.08(\mathrm{~s}, 3 \mathrm{H}) ;{ }^{13} \mathrm{C} \mathrm{NMR}\left(125 \mathrm{MHz}, \mathrm{CDCl}_{3}\right) \delta$ 177.4, 76.3, 75.7, 40.9, 22.9, 18.8; HRMS (CI/NH3) exact mass calcd for $\mathrm{C}_{6} \mathrm{H}_{11} \mathrm{O}_{3}[\mathrm{M}+\mathrm{H}]^{+}, 131.0708$. Found 131.0710. Assay of enantiomeric excess: GC analysis $\left(\beta\right.$-Cyclodextrin, $60{ }^{\circ} \mathrm{C}, 3{ }^{\circ} \mathrm{C} / \mathrm{min}$ at 25 psi, $\mathrm{t}_{\mathrm{r}}($ major $)=16.77, \mathrm{t}_{\mathrm{r}}($ minor $\left.)=17.29\right), 94 \%$ ee.

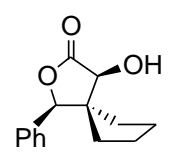

(S,S)-4-Hydroxy-1-phenyl-2-oxa-spiro[4.4]nonan-3-one (Table 2, entry 5). The title compound was prepared according to General Procedure B and isolated as an amorphous white solid (80\% yield). X-ray quality crystals were obtained by recrystallization in hot EtOAc/hexanes: $[\alpha]_{\mathrm{D}}{ }^{25}-14.5^{\circ}\left(c 0.195, \mathrm{CHCl}_{3}\right)$; IR $\left(\mathrm{CHCl}_{3}\right) 3566,3108,2960,2875,2359$, $1784,1456 \mathrm{~cm}^{-1} ;{ }_{1}^{1} \mathrm{H}$ NMR $\left(400 \mathrm{MHz}, \mathrm{CHCl}_{3}\right) \delta 7.42-7.26(\mathrm{~m}, 5 \mathrm{H}), 5.31(\mathrm{~s}, 1 \mathrm{H}), 4.51(\mathrm{~d}, 1 \mathrm{H}, J=3.0 \mathrm{~Hz})$, $2.81(\mathrm{~d}, 1 \mathrm{H}, J=3.2 \mathrm{~Hz}), 2.04-1.89(\mathrm{~m}, 2 \mathrm{H}), 1.71-1.49(\mathrm{~m}, 3 \mathrm{H}), 1.43-1.34(\mathrm{~m}, 1 \mathrm{H}), 1.27-1.20(\mathrm{~m}, 1 \mathrm{H})$, $1.01-0.93(\mathrm{~m}, 1 \mathrm{H}) ;{ }^{13} \mathrm{C} \mathrm{NMR}\left(100 \mathrm{MHz}, \mathrm{CHCl}_{3}\right) \delta 176.9,134.5,128.8,128.6,126.7,84.9,57.2,34.1$, 25.7, 25.0, 24.6; HRMS (EI): Exact mass calcd for $\mathrm{C}_{14} \mathrm{H}_{16} \mathrm{O}_{3}[\mathrm{M}]^{+}, 232.1100$. Found 232.1097. Assay of enantiomeric excess: HPLC analysis (Chiracel AD, $10 \% \mathrm{iPrOH} / \mathrm{hexanes}, 0.7 \mathrm{~mL} / \mathrm{min}, 215 \mathrm{~nm} ; \mathrm{t}_{\mathrm{r}}$ $($ major $)=16.3, \mathrm{t}_{\mathrm{r}}($ minor $\left.)=18.3\right), 99 \%$ ee.

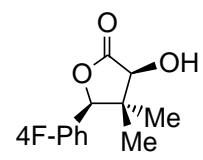

(S,S)-3-Hydroxy-4,4-dimethyl-5-(4-fluorophenyl)-dihydro-furan-2-one (Table 2, entry 6). The title compound was prepared according to General Procedure $\mathrm{B}$ and isolated as a colorless oil (71 \% yield): $[\alpha]_{\mathrm{D}}{ }^{25}-53.0^{\circ}\left(c 0.54, \mathrm{CHCl}_{3}\right)$; IR $\left(\mathrm{CHCl}_{3}\right) 3564$, 3449, 2970, 2917, 1775, 1611, $1514 \mathrm{~cm}^{-1} ;{ }^{1} \mathrm{H}$ NMR (400 MHz, $\left.\mathrm{CHCl}_{3}\right) \delta 7.26(\mathrm{t}, 2 \mathrm{H}$, $J=8.8 \mathrm{~Hz}), 7.09(\mathrm{t}, 2 \mathrm{H}, J=8.8 \mathrm{~Hz}), 5.09(\mathrm{~s}, 1 \mathrm{H}), 4.34(\mathrm{~s}, 1 \mathrm{H}), 1.27(\mathrm{~s}, 3 \mathrm{H}), 0.62(\mathrm{~s}, 3 \mathrm{H}) ;{ }^{13} \mathrm{C} \mathrm{NMR}(100$ $\left.\mathrm{MHz}, \mathrm{CHCl}_{3}\right) \delta 176.8,163.0(\mathrm{~d}, J=248 \mathrm{~Hz}), 129.7,128.0(\mathrm{~d}, J=7.6 \mathrm{~Hz}), 115.6(\mathrm{~d}, J=22.1 \mathrm{~Hz}), 88.5$, 46.5, 22.6, 14.8; HRMS (EI): Exact mass calcd for $\mathrm{C}_{12} \mathrm{H}_{13} \mathrm{O}_{3} \mathrm{~F}[\mathrm{M}]^{+}, 224.0849$. Found 224.0844. 


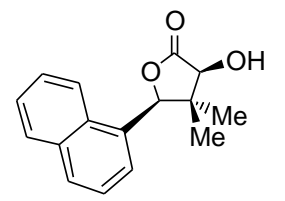

(S,S)-3-Hydroxy-4,4-dimethyl-5-naphthalen-1-yl-dihydro-furan-2-one (Table 2, entry 7). The title compound was prepared according to General Procedure B and isolated as an amorphous pale yellow solid (75\% yield): $[\alpha]_{\mathrm{D}}{ }^{25}-131.0^{\circ}(c 0.61$, $\left.\mathrm{CHCl}_{3}\right)$; IR $\left(\mathrm{CHCl}_{3}\right) 3562,3013,2971,1780,1466,1121 \mathrm{~cm}^{-1} ;{ }^{1} \mathrm{H}$ NMR $(400 \mathrm{MHz}$, $\left.\mathrm{CHCl}_{3}\right) \delta 7.97-7.87(\mathrm{~m}, 3 \mathrm{H}), 7.61(\mathrm{~d}, 1 \mathrm{H}, J=6.6 \mathrm{~Hz}), 7.56-7.51(\mathrm{~m}, 3 \mathrm{H}), 6.10(\mathrm{~s}, 1 \mathrm{H})$, $4.53(\mathrm{~s}, 1 \mathrm{H}), 1.37(\mathrm{~s}, 3 \mathrm{H}), 0.71(\mathrm{~s}, 3 \mathrm{H}) ;{ }^{13} \mathrm{C} \mathrm{NMR}\left(100 \mathrm{MHz}, \mathrm{CHCl}_{3}\right) \delta 177.1,133.9,131.0,129.7$, 129.4, 129.3, 126.6, 125.9, 125.7, 125.3, 123.1, 82.2, 77.9, 47.6, 24.2, 15.7; HRMS (EI): Exact mass calcd for $\mathrm{C}_{16} \mathrm{H}_{16} \mathrm{O}_{3}[\mathrm{M}]^{+}, 256.1099$. Found 256.1100.

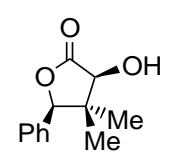

(S,S)-3-Hydroxy-4,4-dimethyl-5-phenyl-dihydro-furan-2-one (Table 2, entry 8). The title compound was prepared according to General Procedure B and isolated as an amorphous white solid (70 \% yield): $[\alpha]_{\mathrm{D}}{ }^{25}-45.1^{\circ}\left(c 0.41, \mathrm{CHCl}_{3}\right)$; IR $\left(\mathrm{CHCl}_{3}\right) 3567,3450$, 3013, 2970, 2934, 2361, 1785, $1774 \mathrm{~cm}^{-1}$; ${ }^{1} \mathrm{H}$ NMR (400 MHz, $\mathrm{CHCl}_{3}$ ) $\delta$ 7.42-7.36 (m, 3H), 7.29-7.26 $(\mathrm{m}, 2 \mathrm{H}), 5.11(\mathrm{~s}, 1 \mathrm{H}), 4.35(\mathrm{~s}, 1 \mathrm{H}), 2.90(\mathrm{br}, 1 \mathrm{H}), 1.29(\mathrm{~s}, 3 \mathrm{H}), 0.63(\mathrm{~s}, 3 \mathrm{H}) ;{ }^{13} \mathrm{C} \mathrm{NMR}(100 \mathrm{MHz}$, $\left.\mathrm{CHCl}_{3}\right) \delta 177.0,133.9,128.8,128.6,126.2,86.0,77.5,46.5,22.7,14.9$; HRMS (EI): Exact mass calcd for $\mathrm{C}_{12} \mathrm{H}_{14} \mathrm{O}_{3}[\mathrm{M}]^{+}$, 206.0943. Found 206.0939.

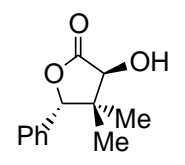

(S,R)-3-Hydroxy-4,4-dimethyl-5-phenyl-dihydro-furan-2-one (Table 2, entry 9). To a dry flask was charged 2-Hydroxy-3,3-dimethyl-4-oxo-4-phenyl-butyric acid ethyl ester (24.2 mg, $0.097 \mathrm{mmol}), \mathrm{Et}_{2} \mathrm{BOMe}(12.6 \mu \mathrm{L}, 0.13 \mathrm{mmol})$, and THF/MeOH (4:1 v/v, $\left.0.7 \mathrm{~mL}\right)$ and aged at RT for $1 \mathrm{~h}$. After cooling to $-78{ }^{\circ} \mathrm{C}, \mathrm{NaBH}_{4}(9.0 \mathrm{mg}, 0.24 \mathrm{mmol})$ was added and the reaction was aged at $-78{ }^{\circ} \mathrm{C}$ for $21 \mathrm{~h}$. To the reaction was added $\mathrm{AcOH}(1 \mathrm{~mL})$. After aging at $\mathrm{rt}$ for $1 \mathrm{~h}$, the mixture was azeotroped in vacuo with $\mathrm{MeOH}\left(3 \times 50 \mathrm{~mL}, 50{ }^{\circ} \mathrm{C}\right)$ and diluted with $\mathrm{Et}_{2} \mathrm{O}$. The mixture was washed with sat. $\mathrm{NaHCO}_{3}$ and then back extracted with $\mathrm{Et}_{2} \mathrm{O}$. The organic layers were combined, dried with $\mathrm{MgSO}_{4}$ and concentrated in vacuo to afford a colorless oil which was transferred to a dry flask and charged with benzene $(1 \mathrm{~mL}), 3 \AA \mathrm{MS}(25 \mathrm{mg})$, and $p$-TSA $(0.5$ equiv). The reaction was stirred at $\mathrm{rt}$ for 30 minutes and diluted with $\mathrm{Et}_{2} \mathrm{O}(4 \mathrm{~mL})$. Triethylamine (6 drops) was added to the mixture, which was then filtered through a plug of silica gel and flushed with $\mathrm{Et}_{2} \mathrm{O}(50 \mathrm{~mL})$. The solvent was removed in vacuo and the residue chromatographed (5-15\% EtOAc/hexanes) to afford the title compound as a colorless oil $(73 \%$ yield $):[\alpha]_{\mathrm{D}}{ }^{25}-58.5^{\circ}\left(c 0.59, \mathrm{CHCl}_{3}\right)$; IR $\left(\mathrm{CHCl}_{3}\right) 3577,3450$, 3018, 2967, 1770, 1605, $1455 \mathrm{~cm}^{-1} ;{ }^{1} \mathrm{H}$ NMR (400 MHz, $\left.\mathrm{CDCl}_{3}\right) \delta$ 7.40-7.32 (m, 3H), 7.18-7.15 (m, $2 \mathrm{H}), 5.28(\mathrm{~s}, 1 \mathrm{H}), 4.21(\mathrm{~d}, 1 \mathrm{H}, J=3.7), 2.91(\mathrm{~d}, 1 \mathrm{H}, J=3.7), 1.26(\mathrm{~s}, 3 \mathrm{H}), 0.81(\mathrm{~s}, 3 \mathrm{H}) ;{ }^{13} \mathrm{C}$ NMR $(100$ $\left.\mathrm{MHz}, \mathrm{CHCl}_{3}\right) \delta 177.3,135.8,128.7,126.0,88.7,75.0,44.0,21.9,20.9$; HRMS (EI): Exact mass calcd for $\mathrm{C}_{12} \mathrm{H}_{14} \mathrm{O}_{3}[\mathrm{M}]^{+}$, 206.0943. Found 206.0942.

\footnotetext{
${ }^{1}$ Pangborn, A. B.; Giardello, M. A.; Grubbs, R. H.; Rosen, R. K.; Timmers, F. J. Organometallics 1996, $15,1518-1520$.

${ }^{2}$ Perrin, D. D. and Armarego, W. L. F. Purification of Laboratory Chemicals; $3^{\text {rd }}$ ed., Pergamon Press, Oxford, 1988.

${ }^{3}$ Manzer, L. E. Inorg. Synth. 1982, 21, 139-140.

${ }^{4}$ Evans, D. A.; Chapman, K. T.; Carreira, E. M. J. Am. Chem. Soc. 1988, 110, 3560-3578.
} 
${ }^{5}$ See preceeding article in this issue for full characterization of compounds.

\section{X-ray crystallographic data for entry 5, table 2.}

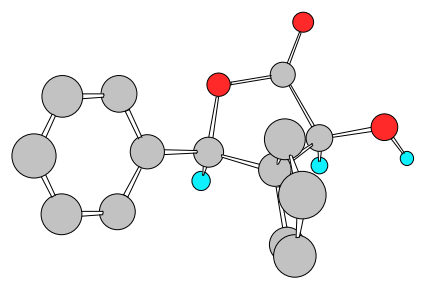

Table 1. Crystal data and structure refinement for JWU2t.

Identification code

Empirical formula

Formula weight

Temperature

Wavelength

Crystal system

Space group

Unit cell dimensions

Volume

Z

Density (calculated)

Absorption coefficient

$\mathrm{F}(000)$

Crystal size

Theta range for data collection

Index ranges

Reflections collected

Independent reflections

Completeness to theta $=28.29^{\circ}$

Absorption correction

Refinement method

Data / restraints / parameters

Goodness-of-fit on $\mathrm{F}^{2}$

Final $\mathrm{R}$ indices [I>2sigma(I)] jwu2t

C14 H16 O3

232.27

293(2) K

$0.71073 \AA$

Orthorhombic

P2(1)2(1)2(1)

$\mathrm{a}=6.1337(8) \AA \quad \alpha=90^{\circ}$.

$\mathrm{b}=7.5487(10) \AA \quad \beta=90^{\circ}$.

$\mathrm{c}=25.784(3) \AA \quad \gamma=90^{\circ}$.

1193.9(3) $\AA^{3}$

4

$1.292 \mathrm{Mg} / \mathrm{m}^{3}$

$0.090 \mathrm{~mm}^{-1}$

496

$.8 \times .8 \times 6.5 \mathrm{~mm}^{3}$

1.58 to $28.29^{\circ}$.

$-7<=\mathrm{h}<=8,-10<=\mathrm{k}<=9,-27<=1<=33$

8021

$2880[\mathrm{R}($ int $)=0.0967]$

$99.0 \%$

None

Full-matrix least-squares on $\mathrm{F}^{2}$

2880 / 0 / 155

1.129

$\mathrm{R} 1=0.0802, \mathrm{wR} 2=0.1823$ 
$\mathrm{R}$ indices (all data)

Absolute structure parameter

Largest diff. peak and hole
$\mathrm{R} 1=0.0889, \mathrm{wR} 2=0.1871$

$-2(2)$

0.310 and -0.309 e. $\AA^{-3}$

Table 2. Atomic coordinates ( $\mathrm{x} 10^{4}$ ) and equivalent isotropic displacement parameters $\AA^{2} \mathrm{x} 10^{3}$ ) for JWU2t. $U(e q)$ is defined as one third of the trace of the orthogonalized $U^{i j}$ tensor.

\begin{tabular}{|c|c|c|c|c|}
\hline & $\mathrm{x}$ & $\mathrm{y}$ & $\mathrm{z}$ & $\mathrm{U}(\mathrm{eq})$ \\
\hline $\mathrm{O}(1)$ & $-2542(4)$ & $3549(3)$ & $9259(1)$ & $36(1)$ \\
\hline $\mathrm{C}(2)$ & $2588(5)$ & $1400(4)$ & $8140(1)$ & $26(1)$ \\
\hline $\mathrm{O}(3)$ & $125(4)$ & $3015(3)$ & $8699(1)$ & $29(1)$ \\
\hline $\mathrm{C}(4)$ & $2166(4)$ & 1987(4) & $8685(1)$ & $24(1)$ \\
\hline $\mathrm{O}(2)$ & $-453(4)$ & $936(3)$ & 9912(1) & $37(1)$ \\
\hline$C(6)$ & $3984(6)$ & $-229(4)$ & $9328(1)$ & $36(1)$ \\
\hline$C(7)$ & $417(5)$ & $-1008(4)$ & $8948(1)$ & $32(1)$ \\
\hline $\mathrm{C}(8)$ & $4675(5)$ & $830(4)$ & $8003(1)$ & $35(1)$ \\
\hline$C(9)$ & $3508(7)$ & $309(5)$ & $7133(1)$ & $43(1)$ \\
\hline$C(10)$ & $-816(5)$ & 2871(4) & $9168(1)$ & $27(1)$ \\
\hline $\mathrm{C}(11)$ & $5114(6)$ & $252(5)$ & $7504(2)$ & $42(1)$ \\
\hline $\mathrm{C}(12)$ & $976(5)$ & 1441(4) & $7762(1)$ & $30(1)$ \\
\hline$C(13)$ & $677(5)$ & $1784(4)$ & $9512(1)$ & $26(1)$ \\
\hline $\mathrm{C}(14)$ & $1435(6)$ & $898(5)$ & $7258(1)$ & $38(1)$ \\
\hline$C(15)$ & $1847(5)$ & $585(4)$ & $9119(1)$ & $23(1)$ \\
\hline$C(16)$ & $1733(11)$ & $-2618(6)$ & $9070(4)$ & $127(4)$ \\
\hline$C(17)$ & $3849(10)$ & $-2165(6)$ & $9219(3)$ & $90(2)$ \\
\hline
\end{tabular}

Table 2. Bond lengths $[\AA]$ and angles $\left[{ }^{\circ}\right]$ for JWU2t.

$\begin{array}{ll}\mathrm{O}(1)-\mathrm{C}(10) & 1.200(4) \\ \mathrm{C}(2)-\mathrm{C}(12) & 1.388(4) \\ \mathrm{C}(2)-\mathrm{C}(8) & 1.396(5) \\ \mathrm{C}(2)-\mathrm{C}(4) & 1.496(4) \\ \mathrm{O}(3)-\mathrm{C}(10) & 1.344(4) \\ \mathrm{O}(3)-\mathrm{C}(4) & 1.473(4)\end{array}$




\begin{tabular}{|c|c|}
\hline $\mathrm{C}(4)-\mathrm{C}(15)$ & $1.551(4)$ \\
\hline $\mathrm{O}(2)-\mathrm{C}(13)$ & $1.396(4)$ \\
\hline$C(6)-C(17)$ & $1.490(6)$ \\
\hline$C(6)-C(15)$ & $1.545(4)$ \\
\hline$C(7)-C(16)$ & $1.493(6)$ \\
\hline $\mathrm{C}(7)-\mathrm{C}(15)$ & $1.552(4)$ \\
\hline $\mathrm{C}(8)-\mathrm{C}(11)$ & $1.385(5)$ \\
\hline $\mathrm{C}(9)-\mathrm{C}(11)$ & $1.374(6)$ \\
\hline$C(9)-C(14)$ & $1.385(5)$ \\
\hline$C(10)-C(13)$ & $1.517(4)$ \\
\hline $\mathrm{C}(12)-\mathrm{C}(14)$ & $1.392(5)$ \\
\hline $\mathrm{C}(13)-\mathrm{C}(15)$ & $1.538(4)$ \\
\hline$C(16)-C(17)$ & $1.396(8)$ \\
\hline $\mathrm{C}(12)-\mathrm{C}(2)-\mathrm{C}(8)$ & $118.8(3)$ \\
\hline $\mathrm{C}(12)-\mathrm{C}(2)-\mathrm{C}(4)$ & $122.0(3)$ \\
\hline $\mathrm{C}(8)-\mathrm{C}(2)-\mathrm{C}(4)$ & $119.2(3)$ \\
\hline $\mathrm{C}(10)-\mathrm{O}(3)-\mathrm{C}(4)$ & $110.1(2)$ \\
\hline $\mathrm{O}(3)-\mathrm{C}(4)-\mathrm{C}(2)$ & $109.0(2)$ \\
\hline $\mathrm{O}(3)-\mathrm{C}(4)-\mathrm{C}(15)$ & $103.6(2)$ \\
\hline$C(2)-C(4)-C(15)$ & $119.7(2)$ \\
\hline$C(17)-C(6)-C(15)$ & $106.1(3)$ \\
\hline$C(16)-C(7)-C(15)$ & $105.4(3)$ \\
\hline $\mathrm{C}(11)-\mathrm{C}(8)-\mathrm{C}(2)$ & $120.7(3)$ \\
\hline $\mathrm{C}(11)-\mathrm{C}(9)-\mathrm{C}(14)$ & $120.4(3)$ \\
\hline $\mathrm{O}(1)-\mathrm{C}(10)-\mathrm{O}(3)$ & $121.5(3)$ \\
\hline $\mathrm{O}(1)-\mathrm{C}(10)-\mathrm{C}(13)$ & $130.4(3)$ \\
\hline $\mathrm{O}(3)-\mathrm{C}(10)-\mathrm{C}(13)$ & 108.2(3) \\
\hline$C(9)-C(11)-C(8)$ & $119.8(3)$ \\
\hline $\mathrm{C}(2)-\mathrm{C}(12)-\mathrm{C}(14)$ & $120.3(3)$ \\
\hline $\mathrm{O}(2)-\mathrm{C}(13)-\mathrm{C}(10)$ & $112.4(3)$ \\
\hline $\mathrm{O}(2)-\mathrm{C}(13)-\mathrm{C}(15)$ & $116.6(2)$ \\
\hline$C(10)-C(13)-C(15)$ & $102.3(2)$ \\
\hline $\mathrm{C}(9)-\mathrm{C}(14)-\mathrm{C}(12)$ & $119.8(3)$ \\
\hline$C(13)-C(15)-C(6)$ & $113.6(2)$ \\
\hline
\end{tabular}




\begin{tabular}{lr}
$\mathrm{C}(13)-\mathrm{C}(15)-\mathrm{C}(4)$ & $97.6(2)$ \\
$\mathrm{C}(6)-\mathrm{C}(15)-\mathrm{C}(4)$ & $114.6(2)$ \\
$\mathrm{C}(13)-\mathrm{C}(15)-\mathrm{C}(7)$ & $112.3(2)$ \\
$\mathrm{C}(6)-\mathrm{C}(15)-\mathrm{C}(7)$ & $105.7(2)$ \\
$\mathrm{C}(4)-\mathrm{C}(15)-\mathrm{C}(7)$ & $113.3(2)$ \\
$\mathrm{C}(17)-\mathrm{C}(16)-\mathrm{C}(7)$ & $111.2(4)$ \\
$\mathrm{C}(16)-\mathrm{C}(17)-\mathrm{C}(6)$ & $110.1(4)$ \\
\hline
\end{tabular}

Symmetry transformations used to generate equivalent atoms:

Table 4. Anisotropic displacement parameters $\left(\AA^{2} \times 10^{3}\right)$ for JWU2t. The anisotropic displacement factor exponent takes the form: $-2 \pi^{2}\left[h^{2} a^{* 2} U^{11}+\ldots+2 h k a^{*} b^{*} U^{12}\right]$

\begin{tabular}{lcccccc}
\hline & $\mathrm{U}^{11}$ & $\mathrm{U}^{22}$ & $\mathrm{U}^{33}$ & $\mathrm{U}^{23}$ & $\mathrm{U}^{13}$ & $\mathrm{U}^{12}$ \\
\hline $\mathrm{O}(1)$ & $39(1)$ & $36(1)$ & $33(1)$ & $3(1)$ & $4(1)$ & $8(1)$ \\
$\mathrm{C}(2)$ & $31(2)$ & $18(1)$ & $29(2)$ & $1(1)$ & $4(1)$ & $-4(1)$ \\
$\mathrm{O}(3)$ & $39(1)$ & $24(1)$ & $23(1)$ & $0(1)$ & $0(1)$ & $5(1)$ \\
$\mathrm{C}(4)$ & $25(1)$ & $20(1)$ & $26(1)$ & $-1(1)$ & $-1(1)$ & $-5(1)$ \\
$\mathrm{O}(2)$ & $45(1)$ & $44(1)$ & $21(1)$ & $6(1)$ & $1(1)$ & $7(1)$ \\
$\mathrm{C}(6)$ & $34(2)$ & $36(2)$ & $38(2)$ & $4(1)$ & $-8(1)$ & $6(1)$ \\
$\mathrm{C}(7)$ & $39(2)$ & $27(1)$ & $30(2)$ & $-2(1)$ & $-1(1)$ & $-9(1)$ \\
$\mathrm{C}(8)$ & $35(2)$ & $35(2)$ & $36(2)$ & $-3(1)$ & $-1(1)$ & $2(1)$ \\
$\mathrm{C}(9)$ & $63(2)$ & $37(2)$ & $28(2)$ & $-11(1)$ & $17(2)$ & $-5(2)$ \\
$\mathrm{C}(10)$ & $36(2)$ & $21(1)$ & $24(1)$ & $-2(1)$ & $1(1)$ & $-7(1)$ \\
$\mathrm{C}(11)$ & $37(2)$ & $43(2)$ & $46(2)$ & $-13(2)$ & $12(2)$ & $2(2)$ \\
$\mathrm{C}(12)$ & $32(2)$ & $30(1)$ & $27(2)$ & $-2(1)$ & $1(1)$ & $-1(1)$ \\
$\mathrm{C}(13)$ & $31(2)$ & $27(1)$ & $21(1)$ & $-4(1)$ & $-4(1)$ & $1(1)$ \\
$\mathrm{C}(14)$ & $51(2)$ & $36(2)$ & $27(2)$ & $-4(1)$ & $-4(2)$ & $-8(2)$ \\
$\mathrm{C}(15)$ & $30(1)$ & $21(1)$ & $19(1)$ & $-1(1)$ & $-3(1)$ & $-4(1)$ \\
$\mathrm{C}(16)$ & $95(4)$ & $25(2)$ & $262(11)$ & $-23(4)$ & $-83(6)$ & $1(2)$ \\
$\mathrm{C}(17)$ & $88(4)$ & $38(2)$ & $145(6)$ & $-2(3)$ & $-51(4)$ & $21(3)$ \\
& & & & & & \\
\hline
\end{tabular}

Table 5. Hydrogen coordinates ( $\left.\mathrm{x} 10^{4}\right)$ and isotropic displacement parameters $\left(\AA^{2} \times 10^{3}\right)$ 
for JWU2t.

\begin{tabular}{lrrrc}
\hline & $\mathrm{x}$ & $\mathrm{y}$ & $\mathrm{z}$ & $\mathrm{U}(\mathrm{eq})$ \\
& & & & \\
$\mathrm{H}(4)$ & 3359 & 2773 & 8790 & 28 \\
$\mathrm{H}(2)$ & 349 & 829 & 10164 & 55 \\
$\mathrm{H}(6 \mathrm{~A})$ & 5235 & 286 & 9154 & 43 \\
$\mathrm{H}(6 \mathrm{~B})$ & 4119 & -19 & 9698 & 43 \\
$\mathrm{H}(7 \mathrm{~A})$ & -949 & -1020 & 9137 & 38 \\
$\mathrm{H}(7 \mathrm{~B})$ & 104 & -947 & 8580 & 38 \\
$\mathrm{H}(8)$ & 5782 & 838 & 8250 & 42 \\
$\mathrm{H}(9)$ & 3815 & -50 & 6796 & 51 \\
$\mathrm{H}(11)$ & 6493 & -173 & 7420 & 50 \\
$\mathrm{H}(12)$ & -417 & 1834 & 7846 & 36 \\
$\mathrm{H}(13)$ & 1756 & 2581 & 9668 & 31 \\
$\mathrm{H}(14)$ & 353 & 930 & 7006 & 46 \\
$\mathrm{H}(16 \mathrm{~A})$ & 1035 & -3272 & 9349 & 153 \\
$\mathrm{H}(16 \mathrm{~B})$ & 1790 & -3380 & 8768 & 153 \\
$\mathrm{H}(17 \mathrm{~A})$ & 4861 & -2472 & 8944 & 108 \\
$\mathrm{H}(17 \mathrm{~B})$ & 4253 & -2826 & 9527 & 108 \\
& & & & \\
\hline
\end{tabular}

Table 6. Torsion angles $\left[^{\circ}\right]$ for JWU2t.

\begin{tabular}{lc}
\hline $\mathrm{C}(10)-\mathrm{O}(3)-\mathrm{C}(4)-\mathrm{C}(2)$ & $151.9(2)$ \\
$\mathrm{C}(10)-\mathrm{O}(3)-\mathrm{C}(4)-\mathrm{C}(15)$ & $23.3(3)$ \\
$\mathrm{C}(12)-\mathrm{C}(2)-\mathrm{C}(4)-\mathrm{O}(3)$ & $-15.7(4)$ \\
$\mathrm{C}(8)-\mathrm{C}(2)-\mathrm{C}(4)-\mathrm{O}(3)$ & $162.9(3)$ \\
$\mathrm{C}(12)-\mathrm{C}(2)-\mathrm{C}(4)-\mathrm{C}(15)$ & $103.2(3)$ \\
$\mathrm{C}(8)-\mathrm{C}(2)-\mathrm{C}(4)-\mathrm{C}(15)$ & $-78.2(4)$ \\
$\mathrm{C}(12)-\mathrm{C}(2)-\mathrm{C}(8)-\mathrm{C}(11)$ & $-2.4(5)$ \\
$\mathrm{C}(4)-\mathrm{C}(2)-\mathrm{C}(8)-\mathrm{C}(11)$ & $179.0(3)$ \\
$\mathrm{C}(4)-\mathrm{O}(3)-\mathrm{C}(10)-\mathrm{O}(1)$ & $-177.1(3)$ \\
$\mathrm{C}(4)-\mathrm{O}(3)-\mathrm{C}(10)-\mathrm{C}(13)$ & $3.4(3)$ \\
$\mathrm{C}(14)-\mathrm{C}(9)-\mathrm{C}(11)-\mathrm{C}(8)$ & $-1.7(6)$
\end{tabular}




$\begin{array}{lc}\mathrm{C}(2)-\mathrm{C}(8)-\mathrm{C}(11)-\mathrm{C}(9) & 2.8(5) \\ \mathrm{C}(8)-\mathrm{C}(2)-\mathrm{C}(12)-\mathrm{C}(14) & 0.9(4) \\ \mathrm{C}(4)-\mathrm{C}(2)-\mathrm{C}(12)-\mathrm{C}(14) & 179.4(3) \\ \mathrm{O}(1)-\mathrm{C}(10)-\mathrm{C}(13)-\mathrm{O}(2) & 25.8(4) \\ \mathrm{O}(3)-\mathrm{C}(10)-\mathrm{C}(13)-\mathrm{O}(2) & -154.7(2) \\ \mathrm{O}(1)-\mathrm{C}(10)-\mathrm{C}(13)-\mathrm{C}(15) & 151.7(3) \\ \mathrm{O}(3)-\mathrm{C}(10)-\mathrm{C}(13)-\mathrm{C}(15) & -28.9(3) \\ \mathrm{C}(11)-\mathrm{C}(9)-\mathrm{C}(14)-\mathrm{C}(12) & 0.2(6) \\ \mathrm{C}(2)-\mathrm{C}(12)-\mathrm{C}(14)-\mathrm{C}(9) & 0.1(5) \\ \mathrm{O}(2)-\mathrm{C}(13)-\mathrm{C}(15)-\mathrm{C}(6) & -76.0(3) \\ \mathrm{C}(10)-\mathrm{C}(13)-\mathrm{C}(15)-\mathrm{C}(6) & 161.0(2) \\ \mathrm{O}(2)-\mathrm{C}(13)-\mathrm{C}(15)-\mathrm{C}(4) & 162.9(2) \\ \mathrm{C}(10)-\mathrm{C}(13)-\mathrm{C}(15)-\mathrm{C}(4) & 39.9(3) \\ \mathrm{O}(2)-\mathrm{C}(13)-\mathrm{C}(15)-\mathrm{C}(7) & 43.8(3) \\ \mathrm{C}(10)-\mathrm{C}(13)-\mathrm{C}(15)-\mathrm{C}(7) & -79.2(3) \\ \mathrm{C}(17)-\mathrm{C}(6)-\mathrm{C}(15)-\mathrm{C}(13) & 129.9(4) \\ \mathrm{C}(17)-\mathrm{C}(6)-\mathrm{C}(15)-\mathrm{C}(4) & -119.1(4) \\ \mathrm{C}(17)-\mathrm{C}(6)-\mathrm{C}(15)-\mathrm{C}(7) & 6.4(5) \\ \mathrm{O}(3)-\mathrm{C}(4)-\mathrm{C}(15)-\mathrm{C}(13) & -38.5(2) \\ \mathrm{C}(2)-\mathrm{C}(4)-\mathrm{C}(15)-\mathrm{C}(13) & -160.1(3) \\ \mathrm{O}(3)-\mathrm{C}(4)-\mathrm{C}(15)-\mathrm{C}(6) & -158.9(2) \\ \mathrm{C}(2)-\mathrm{C}(4)-\mathrm{C}(15)-\mathrm{C}(6) & 79.6(3) \\ \mathrm{O}(3)-\mathrm{C}(4)-\mathrm{C}(15)-\mathrm{C}(7) & 79.8(3) \\ \mathrm{C}(2)-\mathrm{C}(4)-\mathrm{C}(15)-\mathrm{C}(7) & -41.8(4) \\ \mathrm{C}(16)-\mathrm{C}(7)-\mathrm{C}(15)-\mathrm{C}(13) & -123.4(5) \\ \mathrm{C}(16)-\mathrm{C}(7)-\mathrm{C}(15)-\mathrm{C}(6) & 0.9(5) \\ \mathrm{C}(16)-\mathrm{C}(7)-\mathrm{C}(15)-\mathrm{C}(4) & 127.2(5) \\ \mathrm{C}(15)-\mathrm{C}(7)-\mathrm{C}(16)-\mathrm{C}(17) & -8.6(9) \\ \mathrm{C}(7)-\mathrm{C}(16)-\mathrm{C}(17)-\mathrm{C}(6) & \\ \mathrm{C}(15)-\mathrm{C}(6)-\mathrm{C}(17)-\mathrm{C}(16) & \\ & \\ & \end{array}$

Symmetry transformations used to generate equivalent atoms: 